\title{
Spin polarization in the Hubbard model with Rashba spin-orbit coupling on a ladder
}

\author{
José A. Riera \\ Instituto de Física Rosario y Departamento de Física, and Universidad Nacional de Rosario, Rosario, Argentina
}

(Received 10 February 2013; revised manuscript received 27 April 2013; published 1 July 2013)

\begin{abstract}
The competition between on-site Coulomb repulsion and Rashba spin-orbit coupling (RSOC) is studied on two-leg ladders by numerical techniques. By studying persistent currents in closed rings by exact diagonalization, it is found that the contribution to the current due to the RSOC $V_{\mathrm{SO}}$, for a fixed value of the Hubbard repulsion $U$ reaches a maximum at intermediate values of $V_{\text {So }}$. By increasing the repulsive Hubbard coupling $U$, this spin-flipping current is suppressed and eventually it becomes opposite to the spin-conserving current. The main result is that the spin accumulation defined as the relative spin polarization between the two legs of the ladder is enhanced by $U$. Similar results for this Hubbard-Rashba model are observed for a completely different setup in which two halves of the ladders are connected to a voltage bias and the ensuing time-dependent regime is studied by the density matrix renormalization group technique. The combined effect between $V_{\mathrm{SO}}$ and $U$ is also interesting, leading to a strong enhancement of antiferromagnetic order, which in turn may explain the observed behavior of the spin-flipping current. The implications of this enhancement of the spin-Hall effect with electron correlations for spintronic devices is discussed.
\end{abstract}

DOI: 10.1103/PhysRevB.88.045102

PACS number(s): 71.27.+a, 71.70.Ej, 73.23.-b

\section{INTRODUCTION}

Recent trends in the field of spintronics ${ }^{1-3}$ exploit the possibility of controlling electron spins by purely electrical means without using magnetic materials, ${ }^{4}$ which is at the heart of the conceptual proposal of the Datta-Das spin field-effect transistor. ${ }^{5}$ Key to this possibility is the Rashba spin-orbit (SO) interaction ${ }^{6,7}$ appearing due to the structure inversion asymmetry of materials and which has been shown to be tuned by gate voltages in semiconductor heterostructures. ${ }^{8}$

Although the main interest in the study of Rashba spinorbit coupling (RSOC) has been on its ability to generate and control spin-polarized currents, another consequence of the interplay between charge and spin degrees of freedom induced by the RSOC is a flow of spins transversal to the flow of charge, which is known as the spin-Hall effect. ${ }^{9-12}$ If transport takes place along a planar strip, the spin-Hall effect leads to a spin accumulation with opposite projection on both edges of the strip. ${ }^{13,14}$ This has been experimentally observed. ${ }^{15,16}$ Hence, the spin-Hall effect provides a real-space separation between up and down spin electrons, like two ferromagnets with opposite polarizations, thus enhancing the metallic state and its conductance, and these features could also be employed in spintronics devices.

Since most devices and experiments about RSOC have involved semiconductors, electron correlations have mostly not been included so far in theoretical models. However, there are some systems which have been recently considered in this field where such correlations may play an important role. In the first place, RSOC has been observed in oxide heterostructures. ${ }^{17-19}$ The important effects of a RSOC have been experimentally observed in other transition metal oxides, particularly the iridates where the relevant degrees of freedom are the $5 d$ electrons on the $\mathrm{Ir}^{4+}$ ions, and where strong correlation effects are expected. ${ }^{20,21}$ It should be also taken into account that correlation effects become increasingly important in systems with low spatial dimensions. Interesting crossovers have been reported when moving from two-dimensional (2D) systems to quantum wires. $^{22}$ In this sense, spin-orbit effects, predominantly of the Rashba type, have been studied in quantum wires..$^{23,24}$ The presence of RSOC in graphene and carbon nanotubes has been pointed out ${ }^{25}$ and in fact a theoretical study with up to two interacting electrons has been done on a nanotube quantum dot. ${ }^{26}$

In this work, an attempt to study the interplay between electron correlations and RSOC will be performed by considering a Hubbard model with a Rashba SOC. During the last two decades, the difficulties of tackling electron correlations theoretically have been realized. In studies of RSOC, electron correlations have been included only locally regarding quantum dots ${ }^{27,28}$ or on effective one-dimensional (1D) systems using the Tomonaga-Luttinger liquid approach ${ }^{29}$ and conformal field theory. ${ }^{30}$ For this reason, in the present work a two-leg ladder, which may be the narrowest quasi-1D geometry on which a spin-Hall effect could take place, will be considered. The Hubbard-Rashba model on a two-leg ladder is suitable for treatment using essentially exact, unbiased, computational techniques, which have been enormously valuable in the field of strongly correlated electron systems. Particularly for mesoscopic systems, the full quantum manybody interplay between different terms of the Hamiltonian can be reliably captured. There are many compounds that contain two-leg ladder structures such as cuprates, ${ }^{31,32}$ vanadates, ${ }^{33}$ iron chalcogenides, ${ }^{34}$ and other organic and inorganic compounds, although so far the presence of a SOC has not been reported on such materials. It should be also stressed that a Hubbard model in a two-leg ladder has been proposed for carbon nanotubes. ${ }^{35}$ Then, the main goal is to determine and understand the behavior of the spin accumulation as a function of the RSOC and the Hubbard repulsion $U$. The interplay between RSOC and $U$ with respect to a possible metal-insulator transition ${ }^{36}$ will not be discussed in the present work. 


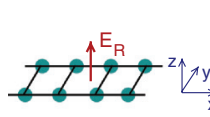

(a)

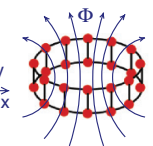

(b)

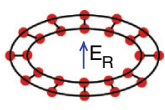

(c)

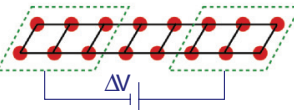

(d)
FIG. 1. (Color online) (a) The two-leg ladder system here considered. (b) A two-leg ring pierced by a magnetic flux. (c) Previously studied 2D rings. (d) Setup used in DMRG calculations.

\section{MODEL AND METHODS}

The Hamiltonian of the Hubbard-Rashba model in a square lattice is defined as:

$$
\begin{aligned}
H= & -t_{0} \sum_{\langle l, m\rangle, \sigma}\left(c_{l, \sigma}^{\dagger} c_{m, \sigma}+\text { H. c. }\right)+U \sum_{l} n_{l, \uparrow} n_{l, \downarrow} \\
& +V_{\mathrm{SO}} \sum_{l}\left[c_{l+x, \downarrow}^{\dagger} c_{l, \uparrow}-c_{l+x, \uparrow}^{\dagger} c_{l, \downarrow}\right. \\
& \left.+i\left(c_{l+y, \downarrow}^{\dagger} c_{l, \uparrow}+c_{l+y, \uparrow}^{\dagger} c_{l, \downarrow}\right)+\text { H. c. }\right],
\end{aligned}
$$

where the notation is standard. The first two terms corresponds to the usual Hubbard model and the last term contains the RSOC. ${ }^{37}$ The couplings $t_{0}$ and $V_{\text {SO }}$ are normalized in such a way that $t_{0}^{2}+V_{\text {SO }}^{2}=1$ (which is henceforth taken as the unit of energy). The longitudinal (transversal) direction of the ladder corresponds to the $x$ axis ( $y$ axis), as shown in Fig. 1(a). The RSOC in Eq. (1) corresponds to an effective Rashba electric field $E_{R}$ along the $z$ axis, i.e., perpendicular to the plane of the ladder. Open boundary conditions (OBC) are applied in the transversal direction. The total number of lattice sites is $N=$ $2 \times L$. Hamiltonian (1) corresponds to the isotropic ladder, in which couplings are the same along both directions. The present study is limited to the quarter-filled system $(n=0.5)$. Weak-coupling analysis ${ }^{38}$ has shown that the Hubbard model on the isotropic ladder at $n=0.5$ is at the boundary between a metal $(C 1 S 0)$ and an insulating $(C 0 S 1)$ phase. ${ }^{39}$ Exactly at the boundary, where additional metallic phases are predicted to be stable, both this study as well as numerical studies ${ }^{40,41}$ and the present results point to a metallic phase, even for the values of $U>0$ here examined. As soon as $V_{\text {SO }}$ is turned on, the occupied bonding band will split leading to a four-point Fermi surface and the system will behave as a metal. In all cases, the length $L$, equal to the total number of electrons, was set equal to $4 m$ ( $m$ being an integer).

To induce persistent currents, a magnetic flux piercing the ring formed by a closed ladder has to be applied [Fig. 1(b)]. This magnetic flux $\Phi$ is included in the Hamiltonian by the usual Peierls factors, which in momentum space is equivalent to replacing $k_{x}$ by $k_{x}+\Phi / L$ (the units in which $e=c=\hbar=1$ have been adopted). These Peierls factors are included in both the hopping and SO terms of Eq. (1). The resulting Hamiltonian is studied using exact diagonalization (ED) for $U>0$. For the noninteracting case, $U=0$, by working in momentum space, ladders with $L$ up to 6400 have been considered. Due to the RSOC the total $S^{z}$ is not conserved, hence all possible values of the total $S^{z}$ have to be included in the Hilbert space. To reduce the Hilbert space dimension, translation symmetry along the longitudinal direction has been implemented thanks to the use of periodic boundary conditions
(PBC) along the $x$ axis. Notice that the ladder ring shown in Fig. 1(b) is different from the 2D ring [Fig. 1(c)] that has been intensively studied in the noninteracting case. ${ }^{42-44}$ Studies including Coulomb interactions for these rings have been limited to a few electrons. ${ }^{45}$

The total current is defined as $J(\Phi)=\frac{\partial E_{0}(\Phi)}{\partial \Phi}$ where $E_{0}(\Phi)$ is the ground state energy. The contribution from the hopping term in Eq. (1), the hopping or spin-conserving current, is then given by the usual current operator along the legs $\hat{j}_{h}=i t_{0} \sum_{l, \sigma}\left(c_{l+\hat{x}, \sigma}^{\dagger} c_{l, \sigma}-\right.$ H. c. $)$. A similar current operator can be easily derived for the contribution from the RSOC term in Eq. (1), that is, the SO or spin-flipping current $\hat{j}_{\text {SO }}$. Notice that $j_{\text {so }}$ turns out to be proportional to $V_{\text {SO }}$. Using the Feynman-Hellmann theorem, the total current is given by $L J=j_{h}+j_{\text {So }}$. In ED calculations, $J, j_{h}$, and $j_{\text {SO }}$ have been independently computed. The spin polarization due to the spin-Hall effect is defined as $\Delta S^{z}=\left\langle S_{1}^{z}-S_{2}^{z}\right\rangle / 2$, where $S_{j}^{z}$ is the total $z$ component of the spin on leg $j$. All physical quantities are ground state averages, and hence, they are functions of the magnetic flux $\Phi$. To determine the effects of the Rashba and Hubbard couplings on these quantities, the reasonable choice of looking at their maximum values as a function of the flux $\Phi$ was adopted.

The second setup considered in this work is shown in Fig. 1(d), which corresponds to OBC along the $x$ axis. Here, at a given time the two ends of a ladder are connected to a voltage bias $\Delta V$ generating a time-dependent regime which is studied by the density matrix renormalization group (DMRG). Although various sophisticated approaches have been proposed within DMRG to deal with this time evolution, ${ }^{46-48}$ the simple "static" approach is implemented in the present work, which is enough to reliably capture the main physical features in the small lattices studied. This is a nonequilibrium process, but a small value $\Delta V=0.01$ was adopted. In this case, the total current is computed as $J(t)=\frac{\partial N_{l}}{\partial t}$, where $N_{l}$ is the total charge in the left half of the ladder, and $t$ is the time measured in units of the time increment $\Delta \tau=0.1 . J_{\max }$ is computed as the average of $J(t)$ between the first two peaks of its time evolution, which can be precisely determined with a relatively small number of retained states $M$ within the static approach. The spin-conserving current $j_{h}(t)$ was computed at the central links of the ladder. $j_{h, \text { max }}$ was computed using the same criterion as for $J_{\max }$. In DMRG calculations, $J$ and $j_{h}$ have been independently computed.

\section{RESULTS}

\section{A. Noninteracting case}

The tight-binding part of the Hamiltonian is, in momentum space,

$$
H_{0}=\sum_{\mathbf{k}}\left(\begin{array}{cccc}
A & B & -t_{\perp} & -i V_{\mathrm{SO}, \perp} \\
B^{*} & A & -i V_{\mathrm{SO}, \perp} & -t_{\perp} \\
-t_{\perp} & i V_{\mathrm{SO}, \perp} & A & B \\
i V_{\mathrm{SO}, \perp} & -t_{\perp} & B^{*} & A
\end{array}\right)
$$

in the basis $\left(c_{1, \mathbf{k}, \uparrow}, c_{1, \mathbf{k}, \downarrow}, c_{2, \mathbf{k}, \uparrow}, c_{2, \mathbf{k}, \downarrow}\right)$, where " 1 " and " 2 " are the two legs of the ladder.

For the general spatially anisotropic ladder

$$
A \equiv-2 t_{\|} \cos k_{x}, \quad B \equiv-2 i V_{\mathrm{SO}, \|} \sin k_{x} .
$$



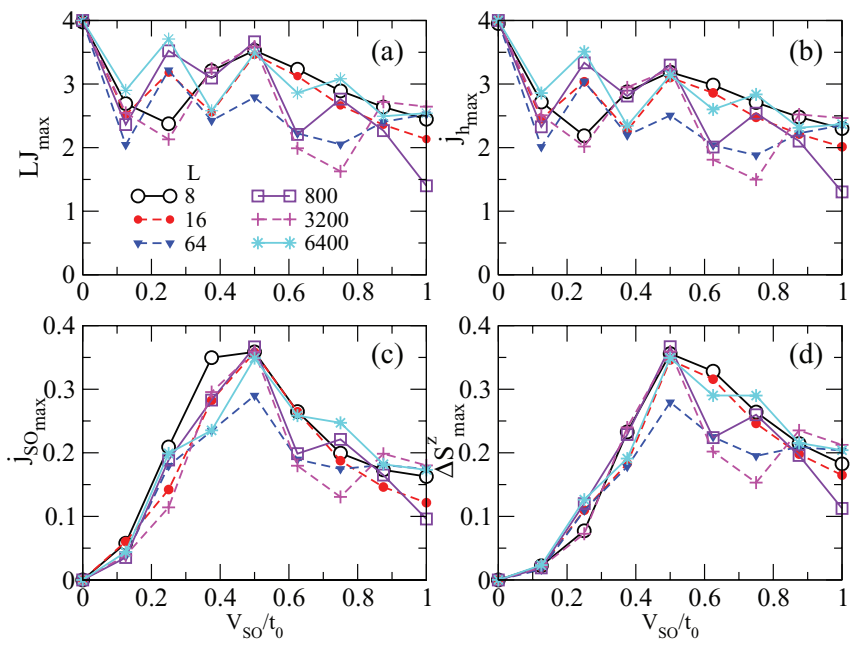

FIG. 2. (Color online) Maximum value of (a) total current, (b) hopping current, (c) spin-orbit current, and (d) spin accumulation $\Delta S^{z}$ as a function of $V_{\mathrm{SO}} / t_{0}$ for the noninteracting system on various two-leg ladders, $n=0.5$. Tight-binding calculations for the persistent currents' setup.

In the isotropic case here considered, $t_{\|}=t_{\perp}=t_{0}, V_{\mathrm{SO}, \|}=$ $V_{\text {SO }, \perp}=V_{\text {SO }}$.

In the tight-binding formalism, the currents $J, j_{h}$, and $j_{\text {so }}$ are independently computed for each value of the magnetic flux $\Phi$, and hence the relation $L J=j_{h}+j_{\text {So }}$ provides an internal check of the calculations.

For the case of persistent currents, Fig. 1(b), results for the noninteracting $U=0$ case are shown in Fig. 2 for various lattice sizes ranging from $L=8$ to 6400 . For the total and the hopping currents [(Figs. 2(a) and 2(b)], a general trend to decrease with increasing $V_{\mathrm{SO}} / t_{0}$ can be observed, although there is a considerable noise due to various level crossings as a function of the flux $\Phi$. In addition it can be observed that the maximum values of the spin-flipping current [Fig. 2(c)] and the spin accumulation [Fig. 2(d)] occur at $V_{\mathrm{SO}} / t_{0} \approx 0.5$. The general decrease of $J_{\max }$ and $j_{h \text {, max }}$ can be explained by a shift of the chemical potential away from the bottom of the antibonding bands [Fig. 3(a)]. The behavior of $j_{\text {SO,max }}$ is more complex and it is determined by the competition between the fact that $V_{\mathrm{SO}}$ is an overall factor in the operator $\hat{j}_{\text {so }}$, and the increase in the shift between the upper and lower spin bands [Fig. 3(a)] with $V_{\text {SO }}$, which works against spin-flipping processes. For $V_{\mathrm{SO}} / t_{0}<0.5$ the band structure does not change too much and $j_{\mathrm{SO} \text {, max }}$ increases approximately linearly with $V_{\mathrm{SO}} / t_{0}$. For $V_{\mathrm{SO}} / t_{0}>0.5$, the band structure changes more strongly and $j_{\text {so,max }}$ starts to be suppressed. The behavior of $\Delta S_{\max }^{z}$ follows that of $j_{\text {SO, max }}$, and it is remarkable that in spite of the noise introduced by level crossings, the ratio $j_{\text {So, } \max } / \Delta S_{\text {max }}^{z}$ follows a smooth monotonous behavior as a function of $V_{\mathrm{SO}} / t_{0}$ for large $L$, as shown in Fig. 3(b).

\section{B. Interacting case}

Let us discuss the correlation effects for the case of persistent currents, for $0 \leqslant U \leqslant 8$. Figure 4 shows the results for the maximum values of the total current $J$, the hopping and spin-orbit contributions to the current $j_{h}$ and $j_{\text {SO }}$, and the
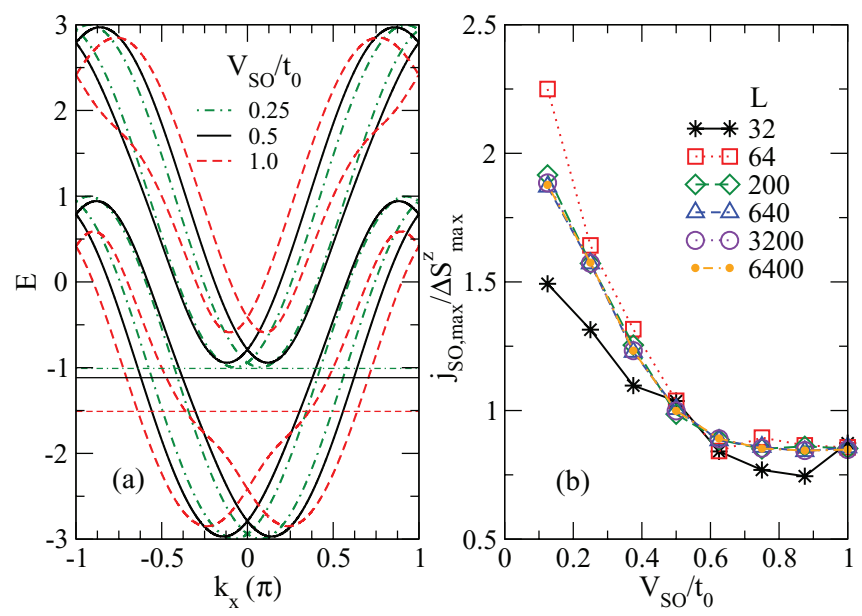

FIG. 3. (Color online) (a) Band structure for various values of $V_{\mathrm{SO}} / t_{0}$. The chemical potentials are indicated with the same convention. (b) Ratio of the maximum values of spin flipping current to the spin accumulation as a function of $V_{\mathrm{SO}} / t_{0}$. Length of the ladder rings are indicated in the plot.

spin-leg polarization $\Delta S^{z}$ on the $2 \times 8$ ladder at $n=0.5$. As can be seen in Fig. 4(a), the total current for a fixed value of $V_{\mathrm{SO}} / t_{0}$ decreases with increasing $U$. This result is expected for $V_{\text {SO }}=0$, i.e., for the pure Hubbard model, where it is well known that the conductance $G$ decreases with increasing $U$, as in the one-dimensional Hubbard model presenting a Luttinger liquid behavior where $G \sim K_{\rho}$ (Ref. 49), where $K_{\rho}$ is the Luttinger exponent that determines the long-range distance behavior of correlations. This behavior was examined for ladders in Ref. 50. The maximum value of the hopping current, shown in Fig. 4(b), follows roughly the same behavior with $U$ and $V_{\mathrm{SO}} / t_{0}$ as the total current since it is much larger than the spin-flipping contribution. The dip in $J$ and $j_{h}$ at $V_{\mathrm{SO}} / t_{0} \approx 0.25$ [Figs. 4(a) and 4(b)] is just a finite-size effect as discussed above. On the other hand, the behavior
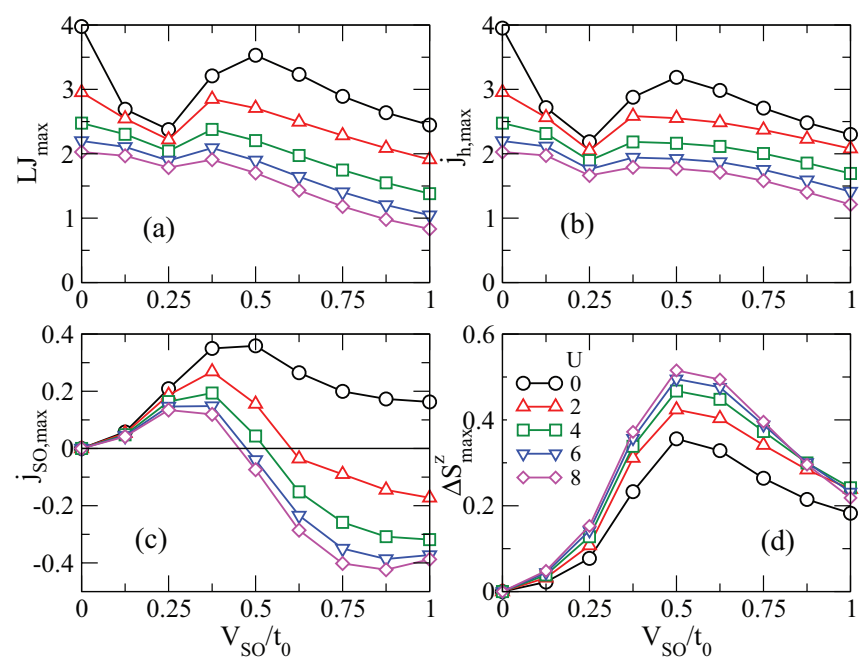

FIG. 4. (Color online) Maximum value of (a) total current, (b) hopping current, (c) spin-orbit current, and (d) spin accumulation $\Delta S^{z}$ as a function of $V_{\mathrm{SO}} / t_{0}$ for various values of $U$. Results for the $2 \times 8$ ladder, $n=0.5$, obtained with ED. 

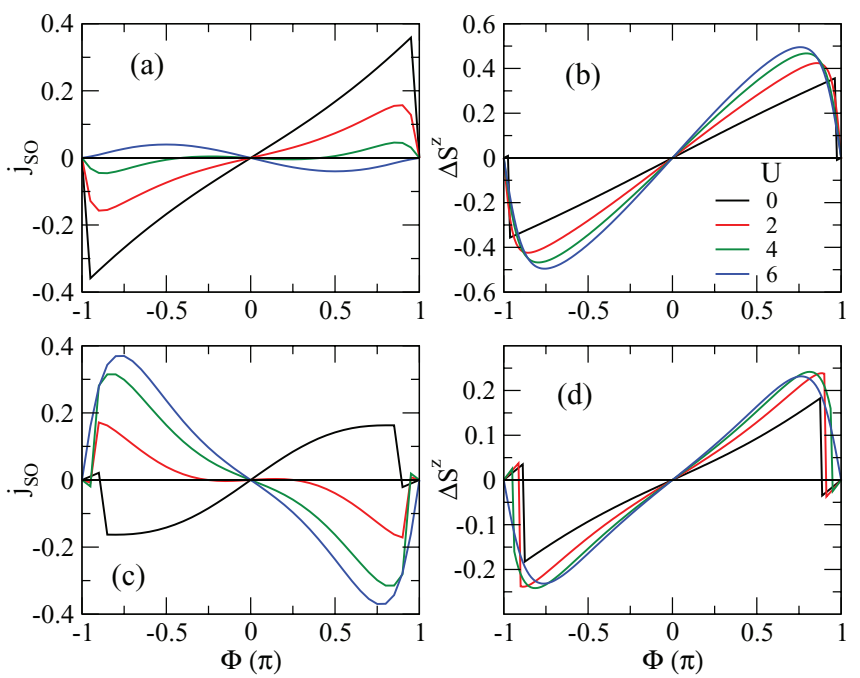

FIG. 5. (Color online) (a) Spin-flipping current and (b) spins accumulation as a function of the magnetic flux for various values of $U$ at $V_{\mathrm{SO}} / t_{0}=0.5$. (c) Spin-flipping current and (d) spin accumulation as a function of the magnetic flux for various values of $U$ at $V_{\mathrm{SO}} / t_{0}=1$. Results for the $2 \times 8$ ladder.

of the maximum value of the SO current is quite interesting [Fig. 4(c)]. For all values of $U, j_{\mathrm{SO} \text {, max }}$ increases with $V_{\mathrm{SO}} / t_{0}$ until reaching a maximum which depends on $U$, and then starts to decrease becoming eventually negative, indicating that its direction is opposed to the hopping current, by convention. The most important results correspond to the maximum value of the spin-leg polarization shown in Fig. 4(d). One should first notice that $\Delta S_{\max }^{z}$ increases as a function of the Coulomb repulsion $U$ for all values of $V_{\mathrm{SO}} / t_{0}$. In addition, its maximum value near $V_{\mathrm{SO}} / t_{0}=0.5$ observed for the noninteracting case is preserved for finite $U$.

The dependence of $j_{\mathrm{SO}}$ and $\Delta S^{z}$ as a function of the magnetic flux, for $V_{\mathrm{SO}} / t_{0}=0.5$ and 1 , is shown in Fig. 5 . Notice that increasing $U$ produces a smooth change in the overall shape of the curve of the flux dependence of the computed quantities, thus justifying the adopted criterion of analyzing their maximum values as a function of $U$ and $V_{\mathrm{SO}} / t_{0}$. In particular, what is interesting in this plot is that the spin-flipping current $j_{\text {so }}$ [Fig. 5(a)] is inverted for $U>4$ for $V_{\mathrm{SO}} / t_{0}=0.5$ thus becoming opposite to the spin-conserving current $j_{h}$, as discussed before. For $V_{\mathrm{SO}} / t_{0}=1$, this feature is observed already for $U=4$ Fig. 5(b).

Results for the voltage bias setup [Fig. 1(d)], for the $2 \times 20$ ladder, are shown in Fig. 6. The behavior of the maximum values of the total, spin-conserving, and spin-flipping currents, and of the spin accumulation as a function of $V_{\mathrm{SO}} / t_{0}$ for various values of $U$ are qualitatively strikingly similar to those shown for the setup involving persistent currents (Fig. 4) in spite of the quite different background physics involved. Notice that actually the maximum values of the currents divided by the voltage bias $\Delta V$ are shown, which correspond to the conductance $G$ up to a factor of $2 \pi$. A comparison with results for the $2 \times 16$ do not show appreciable finite-size effects.

In Fig. 7 some typical time evolutions of the total current and of the spin accumulation are shown for various values of $U$ and $V_{\mathrm{SO}} / t_{0}$. It is well known that the time evolution of the
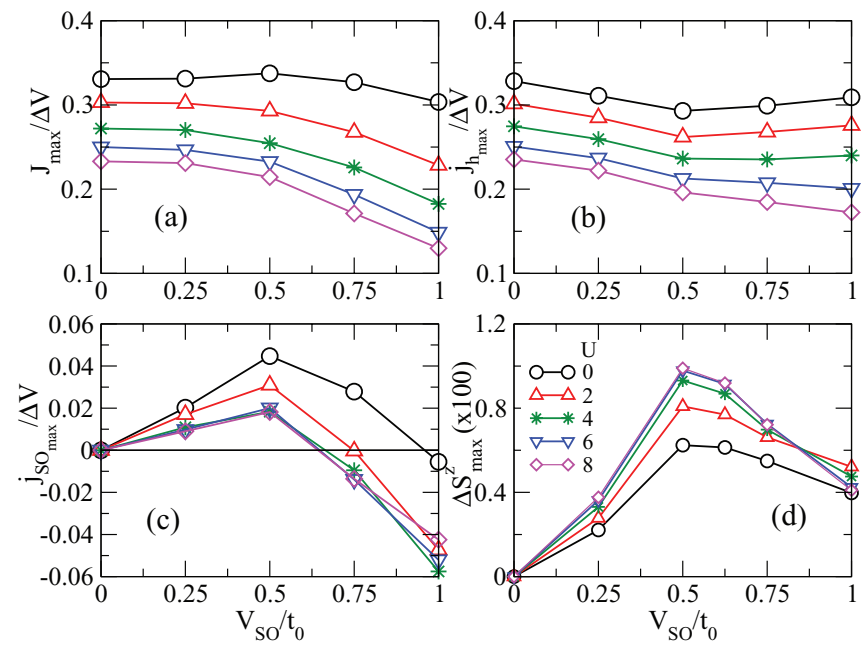

FIG. 6. (Color online) Maximum value of (a) total, (b) hopping, and (c) spin-orbit currents divided by $\Delta V$, and (d) spin accumulation $\Delta S^{z}$ as a function of $V_{\mathrm{SO}} / t_{0}$ for various values of $U$. Results for the $2 \times 20$ ladder, $n=0.5$, obtained with DMRG.

current follows an oscillatory behavior due to the interplay between kinetic and potential energies. As the number of retained states increases, or by adopting an adaptive scheme ${ }^{47}$ in time-dependent DMRG, the current reaches a smooth plateau during each half-period of the time evolution. The value of the current at the plateau divided by the voltage bias $\Delta V$ is equal $G / 2 \pi$, where $G$ is the conductance (remember that units where $e=\hbar=1$ were adopted). In the present case, the adopted criteria of measuring $J_{\max }$ as the average of $J(t)$ between the first two peaks leads to a slight overestimation of $G$ with respect to its exact value at $U=V_{\mathrm{SO}}=0$.
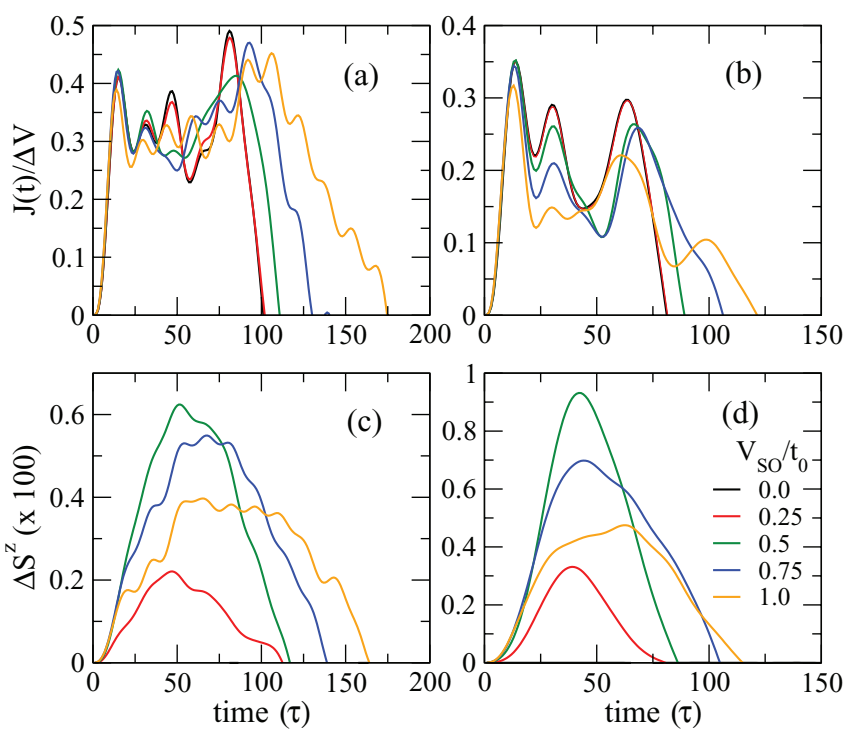

FIG. 7. (Color online) Time evolution of the total current $J$ divided by $\Delta V$ for (a) $U=0$ and (b) $U=4$, and time evolution of the spin accumulation for (c) $U=0$ and (d) $U=4$ for various values of $V_{\mathrm{SO}} / t_{0}$ indicated on the plot. Results for the $2 \times 20$ ladder, obtained by DMRG. Only the first half-period of the time evolution is shown, for the next half-period both $J$ and $\Delta S^{s}$ reverse their signs. 


\section{Magnetic properties}

Some possible explanations of the behaviors shown in Figs. 4 and 6 can be obtained by examining the dependence of various magnetic properties with the parameters $U$ and $V_{\mathrm{SO}} / t_{0}$. $\left(S^{z}\right)^{2}$ and the static magnetic structure factor $S(\mathbf{q})$ are defined as usual from the $z-z$ spin correlations:

$$
\left(S^{z}\right)^{2}=\frac{1}{N} \sum_{j, l}\left\langle\left(S_{j, l}^{z}\right)^{2}\right\rangle, \quad S(\mathbf{q})=\frac{1}{N} \sum_{\mathbf{r}_{1}, \mathbf{r}_{2}}\left\langle S_{\mathbf{r}_{1}}^{z} S_{\mathbf{r}_{2}}^{z}\right\rangle e^{i \mathbf{q} \cdot\left(\mathbf{r}_{1}-\mathbf{r}_{2}\right)}
$$

where the normalization $S_{j, l}^{z}$ equal to $+1(-1)$ for up (down) spin projections was used. With this definition, it is easy to find that for the noninteracting case $\left(S^{z}\right)^{2}=n_{\uparrow}\left(1-n_{\downarrow}\right)+(\uparrow \leftrightarrow \downarrow)$, where $n_{\uparrow}\left(n_{\downarrow}\right)$ is the density of electrons with $\uparrow(\downarrow)$ spin in a state with fixed total $S_{\text {total }}^{z}$. For $S_{\text {total }}^{z}=0,\left(S^{z}\right)^{2}=0.375$, and when $U \rightarrow \infty,\left(S^{z}\right)^{2} \rightarrow n=0.5$ since doubly occupied sites become forbidden.

In Fig. 8(a) the average value of $\left(S^{z}\right)^{2}$, a measure of the magnetic moment per site as a function of $V_{\mathrm{SO}} / t_{0}$ and for various values of $U$ is shown. As expected, $\left(S^{z}\right)^{2}$ increases with $U$, as it is well known for Hubbard-like models, and it is almost independent of $V_{\mathrm{SO}} / t_{0}$. The results for the $2 \times 8$ ladder PBC at zero flux are virtually indistinguishable with the ones obtained for the $2 \times 20$ ladder OBC using DMRG. Note that for $U=0$, one gets the same value as for $S_{\text {total }}^{z}=0$, in spite of the fact that since $S_{\text {total }}^{z}$ is not a quantum number for the model here considered, the ground state is a combination of subspaces with all possible $S_{\text {total }}^{z}$. More interesting are the results for the peak value of the static magnetic structure factor $S^{z z}(\mathbf{q})$, which are shown in Fig. 8(b) as a function of $V_{\mathrm{SO}} / t_{0}$ and for various values of $U$. For large values

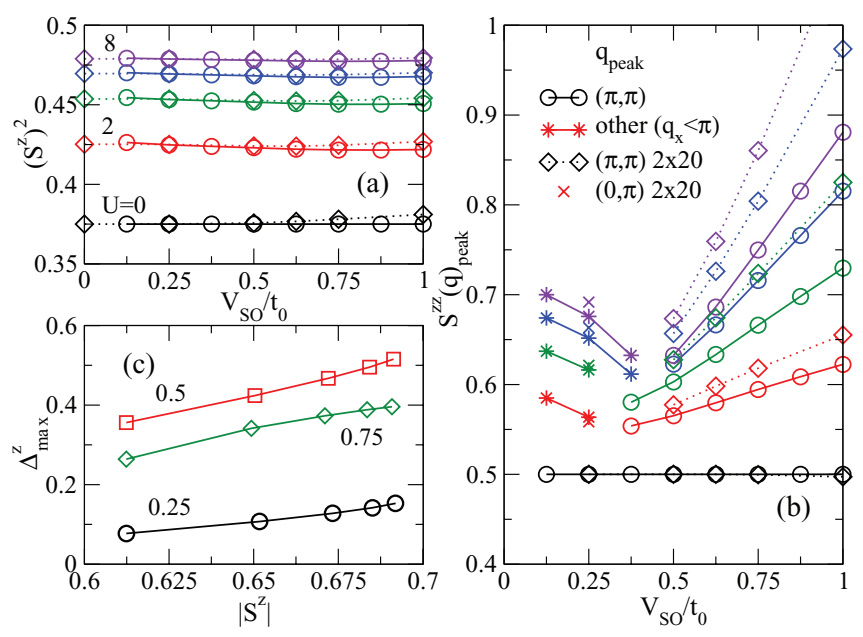

FIG. 8. (Color online) (a) $\left(S^{z}\right)^{2}$ as a function of $V_{\mathrm{SO}} / t_{0}$ for $U=$ $0,2,4,6,8$ from bottom to top for the $2 \times 8$ ladder PBC at zero flux (circles) and for the $2 \times 20$ ladder OBC (diamonds). (b) Peak value of the magnetic structure factor $S^{z z}(\mathbf{q})$ as a function of $V_{\mathrm{SO}} / t_{0}$ for $U=0,2,4,6,8$ from bottom to top for the $2 \times 8$ ladder PBC at zero flux (circles, stars) and for the $2 \times 20$ ladder OBC (diamonds, crosses) if $\mathbf{q}=(\pi, \pi)$ (other). (c) Maximum value of the spin accumulation $\Delta S^{z}$ as a function of $\left|S^{z}\right|$ at zero flux for various values of $V_{\mathrm{SO}} / t_{0}$ indicated in the plot for the $2 \times 8$ ladder PBC. of $V_{\mathrm{SO}} / t_{0}, S^{z z}(\mathbf{q})$ has a peak at $\mathbf{q}=(\pi, \pi)$, while for small values of $V_{\mathrm{SO}} / t_{0}, S^{z z}(\mathbf{q})$ is maximal at other values of $\mathbf{q}$, all of them with $q_{x} \neq \pi$, and in most cases at $\mathbf{q}=(0, \pi)$. In the first place, as in Fig. 8(a), it is expected that $S^{z z}(\mathbf{q})$ is enhanced by $U$. What is unexpected is the strong dependence of $S^{z z}(\mathbf{q})$ with $V_{\mathrm{SO}} / t_{0}: S^{z z}(\pi, \pi)$ increases with increasing $V_{\mathrm{SO}} / t_{0}$, and $S^{z z}[\mathbf{q} \neq(\pi, \pi)]$ increases with decreasing $V_{\mathrm{SO}} / t_{0}$. This is a combined effect of $V_{\mathrm{SO}} / t_{0}$ and $U$ since for $U=0$, $S^{z z}(\mathbf{q})$ is constant and clearly the slope of $S^{z z}(\mathbf{q})$ with $V_{\mathrm{SO}} / t_{0}$ increases with $U$. The results for the $2 \times 20$ ladder show a stronger dependence than the ones corresponding to the $2 \times 8$ ladder and this could be explained by the fact that in DMRG $S^{z z}(\mathbf{q})$ calculations, the only included spin-spin correlations are the ones measured from one of the central sites of the ladder.

The increase of the $z$ component of the magnetic moment can at least partially explain the larger values of the spin accumulation observed as a function of the Hubbard repulsion for a fixed value of $V_{\mathrm{SO}} / t_{0}$. This is shown in Fig. 8(c) where it can be observed an almost linear dependence of $\Delta S_{\max }^{z}$ with $\left|S^{z}\right|$ which is expected for an density operator. Of course, the dependence of the slope and $U=0$ values with $V_{\mathrm{SO}} / t_{0}$ is not trivial. The peak of the magnetic structure factor at $\mathbf{q}=(\pi, \pi)$, indicating the presence of an antiferromagnetic (AFM) order could in turn provide some insight on the suppression of the spin-flipping current $j_{\mathrm{SO}}$ for large $V_{\mathrm{SO}} / t_{0}$ and $U$, shown in Figs. 4(c) and 6(c). For an AFM order, two electrons occupying nearest-neighbors sites along one leg of the ladder would likely have spins with opposite projections on the $z$ axis. For this configuration, a spinflipping hopping would be impossible while there would be no problem for a spin-conserving hopping (except for a cost $U)$. Now, once a $t_{0}$ hopping has occurred, creating a doubly occupied site and leaving behind an empty site, a $V_{\text {SO }}$ hopping is now possible from the doubly occupied site to the empty one. Hence the spin-flipping hopping takes place in a direction opposite to the spin-conserving one, thus possibly explaining the observed change of sign of the spin-flipping current.

\section{CONCLUSION}

To summarize, we have considered perhaps the simplest system where some features associated to Rashba spin-orbit coupling can be observed. ${ }^{14}$ This two-leg ladder system is suitable to be studied by unbiased, essentially exact, computational techniques, which allow to consider electron correlations on an equal footing with spin-conserving and spin-flipping hoppings. At quarter-filling it was observed that spin accumulation is maximal at an intermediate value of $V_{\mathrm{SO}} / t_{0}$, independently of $U$. By increasing a repulsive $U$, the spin-flipping current is suppressed and it becomes negative for large $V_{\text {SO }}$ and $U$, while spin accumulation is enhanced. These two last features can be explained by the increase of $\left(S^{z}\right)^{2}$ and AFM correlations, the latter being a combined effect of $V_{\text {So }}$ and $U$. The present approach could be extended to wider strips which could in principle be studied by DMRG, and also by variational and diffusion Monte Carlo (plus a fixed-node approximation), and to different electron fillings. In addition, some subtle and so far controversial properties, such as spin 
currents, could be studied numerically on this class of strips including electron correlations. Although the present study does not include any dissipative mechanism, an enhancement of spin accumulation by electron interactions together with a reduction of the overall current are positive features that could be taken advantage in spintronic devices.

\section{ACKNOWLEDGMENTS}

The author wishes to acknowledge Sadamichi Maekawa for calling his attention to related problems and to George Martins for a careful reading of the manuscript. The author is supported in part by the Consejo Nacional de Investigaciones Científicas y Técnicas, Argentina.
${ }^{1}$ S. A. Wolf, D. D. Awschalom, R. A. Buhrman, J. M. Daughton, S. von Molnar, M. L. Roukes, A. Y. Chtchelkanova, and D. M. Treger, Science 294, 1488 (2001).

${ }^{2}$ G. A. Prinz, Science 282, 1660 (1998).

${ }^{3}$ I. Zutic, J. Fabian, and S. Das Sarma, Rev. Mod. Phys. 76, 323 (2004).

${ }^{4}$ D. Awschalom, Physics 2, 50 (2009).

${ }^{5}$ S. Datta and B. Das, Appl. Phys. Lett. 56, 665 (1989).

${ }^{6}$ E. I. Rashba, Sov. Phys. Solid State 2, 1109 (1960); Y. A. Bychkov and E. I. Rashba, Sov. Phys. JETP Lett. 39, 78 (1984).

${ }^{7}$ For a review see, R. Winkler, Spin-Orbit Coupling Effects in Two-Dimensional Electron and Hole Systems (Springer, New York, 2003).

${ }^{8}$ J. B. Miller, D. M. Zumbuhl, C. M. Marcus, Y. B. Lyanda-Geller, D. Goldhaber-Gordon, K. Campman, and A. C. Gossard, Phys. Rev. Lett. 90, 076807 (2003).

${ }^{9}$ M. I. D'yakonov and V. I. Perel, Sov. Phys. JETP 33, 1053 (1971).

${ }^{10}$ J. E. Hirsch, Phys. Rev. Lett. 83, 1834 (1999).

${ }^{11}$ S. Murakami, N. Nagaosa, and S.-C. Zhang, Science 301, 1348 (2003).

${ }^{12}$ J. Sinova, D. Culcer, Q. Niu, N. A. Sinitsyn, T. Jungwirth, and A. H. MacDonald, Phys. Rev. Lett. 92, 126603 (2004).

${ }^{13}$ B. K. Nikolić, S. Souma, L. P. Zârbo, and J. Sinova, Phys. Rev. Lett. 95, 046601 (2005).

${ }^{14}$ A. G. Malshukov, L. Y. Wang, C. S. Chu, and K. A. Chao, Phys. Rev. Lett. 95, 146601 (2005).

${ }^{15}$ Y. Kato, R. C. Myers, A. C. Gossard, and D. D. Awschalom, Science 306, 1910 (2004).

${ }^{16} \mathrm{~J}$. Wunderlich, B. Kaestner, J. Sinova, and T. Jungwirth, Phys. Rev. Lett. 94, 047204 (2005).

${ }^{17}$ A. D. Caviglia, M. Gabay, S. Gariglio, N. Reyren, C. Cancellieri, and J.-M. Triscone, Phys. Rev. Lett. 104, 126803 (2010).

${ }^{18}$ H. Nakamura, T. Koga, and T. Kimura, Phys. Rev. Lett. 108, 206601 (2012).

${ }^{19}$ P. D. C. King, R. H. He, T. Eknapakul, P. Buaphet, S.-K. Mo, Y. Kaneko, S. Harashima, Y. Hikita, M. S. Bahramy, C. Bell, Z. Hussain, Y. Tokura, Z.-X. Shen, H. Y. Hwang, F. Baumberger, and W. Meevasana, Phys. Rev. Lett. 108, 117602 (2012).

${ }^{20}$ B. J. Kim, H. Jin, S. J. Moon, J.-Y. Kim, B.-G. Park, C. S. Leem, J. Yu, T. W. Noh, C. Kim, S.-J. Oh, J.-H. Park, V. Durairaj, G. Cao, and E. Rotenberg, Phys. Rev. Lett. 101, 076402 (2008).

${ }^{21}$ M. Trescher and E. J. Bergholtz, Phys. Rev. B 86, 241111 (2012).

${ }^{22}$ P. Wenk and S. Kettemann, Phys. Rev. B 83, 115301 (2011).
${ }^{23}$ C. H. L. Quay, T. L. Hughes, J. A. Sulpizio, L. N. Pfeiffer, K. W. Baldwin, K. W. West, D. Goldhaber-Gordon, and R. de Picciotto, Nat. Phys. 6, 336 (2010).

${ }^{24}$ A. E. Hansen, M. T. Bjork, I. C. Fasth, C. Thelander, and L. Samuelson, Phys. Rev. B 71, 205328 (2005).

${ }^{25}$ D. Huertas-Hernando, F. Guinea, and A. Brataas, Phys. Rev. B 74, 155426 (2006); Phys. Rev. Lett. 103, 146801 (2009); J.-S. Jeong, J. Shin, and H.-W. Lee, Phys. Rev. B 84, 195457 (2011).

${ }^{26}$ B. Wunsch, Phys. Rev. B 79, 235408 (2009).

${ }^{27}$ D. F. Mross and H. Johannesson, Phys. Rev. B 80, 155302 (2009).

${ }^{28}$ Q.-F. Sun, J. Wang, and H. Guo, Phys. Rev. B 71, 165310 (2005).

${ }^{29}$ M. Pletyukhov and V. Gritsev, Phys. Rev. B 70, 165316 (2004).

${ }^{30}$ A. A. Zvyagin, Phys. Rev. B 86, 085126 (2012).

${ }^{31}$ M. Azuma, Z. Hiroi, M. Takano, K. Ishida, and Y. Kitaoka, Phys. Rev. Lett. 73, 3463 (1994).

${ }^{32}$ M. Uehara, T. Nagata, J. Akimitsu, H. Takahashi, N. Mori, and K. Kinoshita, J. Phys. Soc. Jpn. 65, 2764 (1996).

${ }^{33}$ D. Smirnov, P. Millet, J. Leotin, D. Poilblanc, J. Riera, D. Augier, and P. Hansen, Phys. Rev. B 57, R11035 (1998).

${ }^{34}$ J. M. Caron, J. R. Neilson, D. C. Miller, A. Llobet, and T. M. McQueen, Phys. Rev. B 84, 180409(R) (2011).

${ }^{35}$ L. Balents and M. P. A. Fisher, Phys. Rev. B 55, R11973 (1997).

${ }^{36}$ D. A. Pesin and L. Balents, Nat. Phys. 6, 376 (2010).

${ }^{37}$ T. P. Pareek and P. Bruno, Phys. Rev. B 65, 241305 (2002).

${ }^{38}$ L. Balents and M. P. A. Fisher, Phys. Rev. B 53, 12133 (1996).

${ }^{39}$ J. Riera, D. Poilblanc, and E. Dagotto, Eur. J. Phys. B 7, 53 (1999).

${ }^{40}$ R. M. Noack, S. R. White, and D. J. Scalapino, Physica C 270, 281 (1996).

${ }^{41}$ M. Vojta, A. Hübsch, and R. M. Noack, Phys. Rev. B 63, 045105 (2001).

${ }^{42}$ Xin Liu, M. F. Borunda, Xiong-Jun Liu, and J. Sinova, Phys. Rev. B 80, 174524 (2009).

${ }^{43}$ J. S. Sheng and Kai Chang, Phys. Rev. B 74, 235315 (2006).

${ }^{44}$ J. Splettstoesser, M. Governale, and U. Zulicke, Phys. Rev. B 68, 165341 (2003)

${ }^{45}$ C. Daday, A. Manolescu, D. C. Marinescu, and V. Gudmundsson, Phys. Rev. B 84, 115311 (2011).

${ }^{46}$ A. E. Feiguin and S. R. White, Phys. Rev. B 72, 020404 (2005).

${ }^{47}$ U. Schollwöck, Rev. Mod. Phys. 77, 259 (2005).

${ }^{48}$ P. Schmitteckert, Phys. Rev. B 70, 121302(R) (2004).

${ }^{49}$ C. L. Kane and M. P. A. Fisher, Phys. Rev. B 46, 7268 (1992).

${ }^{50}$ E. Orignac and T. Giamarchi, Phys. Rev. B 56, 7167 (1997). 